\title{
Die Praxis der ambulanten Zwangsbehandlung in anderen Ländern
}

\author{
Welchen Stellenwert nehmen ambulante psychiatrische Zwangsmassnahmen in ver- \\ schiedenen Ländern ein? Die Analyse internationaler Studienresultate ergibt, dass \\ sich gegenüber der Standardbehandlung keine eindeutigen Vorteile finden lassen. \\ Vor diesem Hintergrund wirft die Absicht, ambulante psychiatrische Zwangsmass- \\ nahmen in der Schweiz neu zuzulassen, berechtigte Fragen auf und sollte kritisch \\ diskutiert werden.
}

René Bridler ${ }^{a}$

Jürg Gassmann ${ }^{b}$

a Dr. med., FMH Psychiatrie und Psychotherapie, Ärztlicher Direktor Sanatorium Kilchberg AG, Privatklinik für Psychiatrie und Psychotherapie

b Rechtsanwalt, ehemaliger Geschäftsleiter von Pro Mente Sana
* Teil 1: Bridler R, Gassmann J. Das neue Erwachsenenschutzrecht - wichtige Änderungen für die Psychiatrie.

Schweiz Ärztezeitung. 2010;91(44):1749-52.

Korrespondenzen: Dr. med. René Bridler Sanatorium Kilchberg AG Alte Landstrasse 70 CH-8802 Kilchberg Tel. 0447164265 Fax 0447164343

r.bridler@

sanatorium-kilchberg.ch

Jürg Gassmann

Zürcherstrasse 41 Gebäude 100 CH-8400 Winterhur

Tel. 0522627043

info@gassmannlaw.ch
Nach ihrer Einführung in den USA in den 1960er und 70er Jahren haben sich in den meisten angelsächsischen Ländern und in mehreren EU-Staaten Formen der ambulanten Zwangsbehandlung etabliert. Die ersten Programme in den USA waren im Zuge der damaligen Kritik an der durchwegs als repressiv empfundenen Krankenhauspsychiatrie entstanden und wurden als Möglichkeit gesehen, Klinikaufenthalte zu vermeiden und die Betroffenen mit einer zwar unfreiwilligen, aber weniger einschneidenden Massnahme in ihrem gewohnten Lebensumfeld gemeindenah zu behandeln (less restrictive environment). In einer zweiten Phase wurden ambulante Zwangsmassnahmen vermehrt als geeignete Massnahmen zur Eindämmung der «Drehtürpsychiatrie» sowie zum Schutz der Gesellschaft vor wiederkehrender Fremd- und Selbstgefährdung bei behandlungsunwilligen Patienten gefordert. Dabei ist bemerkenswert, dass einzelne Staaten eine entsprechende Gesetzgebung im Anschluss an ein medienwirksames Tötungsdelikt durch eine psychisch kranke Person in Gang setzten [1].

In der Literatur steht der Begriff der ambulanten Zwangsbehandlung (engl. compulsory community treatment, involuntary outpatient treatment) für eine ganze Reihe von Massnahmen bzw. Therapieoptionen, die teilweise auch nebeneinander existieren. Auf einen gemeinsamen Nenner gebracht, ist damit immer eine gesetzliche Regelung von staatlich durchsetzbaren psychiatrischen Behandlungen in einem ambulanten Setting ohne notwendige Verknüpfung mit einem vorangegangenen Klinikaufenthalt gemeint [4]. Die Massnahmen selbst unterscheiden sich aber von Land zu Land beträchtlich. Es gibt Länder, welche die verzögerte bzw. überwachte Entlassung aus der Klinik kennen mit Auflagen zur Nachbehandlung (extended release, supervised discharge) und der Möglichkeit, die betroffene Person bei Non-Compliance präventiv in das stationäre Setting zurückzuversetzen. Andere

\section{Résumé}

Le nouveau droit de la protection des adultes, planifié d'entrer en vigueur dans l'année 2013 ou 2014, prévoit la possibilité des traitements médicaux ambulatoires obligatoires administrés contre la volonté de la personne concernée - faisant une réserve expresse en faveur du droit cantonal. Le nouveau droit concerne en première ligne la psychiatrie. La première partie de l'article, paru dans le numéro 44 du BMS* a éclairé le cadre légal, les options thérapeutiques ainsi que des arguments courants en faveur de la prise en charge en ambulatoire de façon obligatoire. La deuxième partie ici-présente donne une indication brève sur la littérature empirique concernant ce sujet et traite des arguments contre des mesures ambulatoires. Quelques propres conclusions critiques complètent ce papier.**

** Wir danken Frau Dr. med. Heike Russmann für die Übersetzung.

ermöglichen ambulante Zwangsbehandlungen mit dem Ziel, Hospitalisationen zu vermeiden und frühe Klinikentlassungen zu erleichtern im Sinn des Least restrictive environment (s. oben). Zur Sicherstellung der Kontakte sind mancherorts polizeiliche Zuführungen möglich, eine zwangsweise Verabreichung von Psychopharmaka ist dagegen in den meisten Ländern nicht vorgesehen oder explizit verboten; dort, wo diese Praxis erlaubt wäre, wie beispielsweise in Neuseeland, 
wird sie aber kaum je angewandt, da sie von den Behandelnden gemeinhin als gefährlich und unethisch abgelehnt wird $[2,5]$. Im weiteren gibt es beträchtliche Unterschiede bezüglich der Anordnung, Dauer und Überprüfung von Massnahmen sowie ihrer Verknüpfung mit vorausgegangenen stationären Aufenthalten. In einzelnen Ländern können den Patienten zusätzlich oder alternativ zur Zwangsbehandlung eine bestimmte Arbeits- und Wohnform, Einschränkungen der Reisefreiheit und eine Gewährung des Wohnungszutritts durch den verantwortlichen Therapeuten auferlegt werden [1].

\section{Eine zwangsweise Verabreichung von Psychopharmaka ist in den meisten Ländern nicht vorgesehen oder explizit verboten}

zwangsweise Verabreichung von Psychopharmaka ist jedoch explizit untersagt. Das Resultat der Metaanalyse war ernüchternd, da sich keine Hinweise auf eine überlegene Wirksamkeit der OPC fanden hinsichtlich Psychopathologie, Spitalaufenthalte, Verhaftungen, Obdachlosigkeit und Behandlungszufriedenheit. Überraschenderweise wurden die OPC-Patienten jedoch signifikant weniger häufig selber Opfer von Verbrechen. In NNT (numbers needed to treat) ausgedrückt, wäre jeweils folgende Anzahl von OPC notwendig, um ein einziges der folgenden Ereignisse zu verhindern: 85 für eine Spitaleinweisung, 27 für eine Obdachlosigkeit und 238 für eine Verhaftung [6].

Dieselben Autoren unterzogen auch die nicht randomisierten Studien mit einer Kontrollgruppe einer systematischen Review und orientierten sich dabei an den Empfehlungen der Cochrane Effective Practice and Organizational Change (EPOC) group [7]. Sie schlossen drei Untersuchungen mit einem VorherNachher-Design in ihre Analyse mit ein. In beiden Gruppen (Intervention und Kontrolle) kam es in der zweiten Periode zu einer signifikanten Reduktion der Spitaltage, die Patienten in der Interventionsgruppe hatten aber signifikant weniger Spitalaufenthalte, die länger als drei Monate dauerten. Bezüglich Hospitalisationsrate unterschieden sich die Gruppen nicht voneinander, die Patienten der Interventionsgruppe wurden jedoch rascher in die Klinik eingewiesen, was auf eine erhöhte therapeutische Wachsamkeit hinweist und nicht negativ interpretiert werden muss. In zwei Studien fand sich bezüglich Adherence zur Behandlung kein Unterschied, in der dritten Untersuchung kam es bei den Massnahmepatienten zu einer Steigerung von insgesamt fünf Therapiekontakten pro Jahr im Vergleich zur Vorperiode [7].

Zahlreiche naturalistische, meist kleinere Untersuchungen ergaben zumeist Vorteile zugunsten der ambulanten Zwangsbehandlung wie beispielsweise eine Zunahme von Therapiekontakten oder eine Reduktion der Spitaltage, da die Massnahmepatienten früher entlassen werden konnten. Übereinstimmend fanden aber auch sie keine Verringerung oder sogar eine Zunahme der Anzahl der stationären Aufenthalte. Allerdings verfügten diese Studien oftmals nicht über spezifizierte Einschlusskriterien und nur teilweise über ein Kontrollgruppendesign und sind daher anfällig für Störgrössen und Fehlinterpretationen [1, 6, 7].

Kritische Stimmen mahnen davor, die negativen Resultate der grossen Studien und Metaanalysen zu stark zu gewichten. Die Anwendung der traditionellen Hierarchie der evidenzbasierten Medizin werde komplexen gemeindenahen, auf lokale Gegebenheiten Rücksicht nehmenden sozial-medizinischen Interventionen nicht gerecht. Auffällig sei zudem eine internationale bzw. interkulturelle Konvergenz des Profils derjenigen Patienten, bei denen eine Zwangsmassnahme angewandt werde. Es handle sich dabei typischerweise um ca. 40-jährige Männer mit einer langen, von stationären Aufenthalten geprägten Vorgeschichte 
einer psychotischen Erkrankung [5]. Es brauche daher andere Methoden, um Wirkung und Akzeptanz von ambulanten Zwangsmassnahmen fair beurteilen zu können wie beispielsweise gezielte Befragungen von beteiligten Patienten und Klinikern.

Gibbs et al. [2] identifizierten in der Provinz Otago (Neuseeland) 103 Patienten, bei denen in den vergangenen zwei Jahren während mindestens 6 Monaten eine Zwangsbehandlung durchgeführt worden war und bei denen es nicht zu einer Rehospitalisation gekommen war (das Gesamttotal der Personen mit einer Zwangsbehandlung wird nicht berichtet). Von den 103 Patienten wurden 19 von ihren Betreuern als zur Befragung unfähig beurteilt und schieden aus. Von den verbleibenden 84 Personen partizipierte genau die Hälfte ( $n=42$, entspricht $41 \%$ der ursprünglich 103 Patienten) an der Untersuchung. 65\% der Inter- trollierten Untersuchungen und drei robusten kontrollierten Studien mit einem Vorher-Nachher-Design keine eindeutigen Vorteile von ambulanten psychiatrischen Zwangsmassnahmen im Vergleich mit der jeweiligen Standardbehandlung zeigt. Insbesondere lässt sich nicht bestimmen, ob einzelne Vorteile tatsächlich auf den Zwangscharakter der Massnahme oder nicht eher auf das intensivierte Betreuungsangebot zurückzuführen sind. Dies lässt sich insbesondere dort vermuten, wo sowohl bei der Kontroll- als auch bei der Interventionsgruppe eine Reduktion der Spitaltage zu beobachten war [7]. Stark in Frage gestellt scheint auch die Erwartung, dass ambulanter Zwang die Drehtürpsychiatrie zu reduzieren vermöge [11]. Allerdings muss festgehalten werden, dass die Generalisierbarkeit der dargestellten Resultate eingeschränkt ist. Beispielsweise wurden bei beiden rando-

\section{Die Literatur zeigt keine eindeutigen Vorteile von ambulanten psychiatrischen Zwangsmassnahmen im Vergleich mit der jeweiligen Standardbehandlung}

viewten beurteilten die laufende oder bereits abgeschlossene Zwangsbehandlung als sehr vorteilhaft oder vorteilhaft, während nur $14 \%$ sie ganz oder mehrheitlich ablehnten. Die Patienten empfanden im allgemeinen die ambulante Zwangsbehandlung als weniger einschneidend im Vergleich mit Obdachlosigkeit, einer Langzeithospitalisation oder einem Gefängnisaufenthalt. Verschiedene Patienten gaben an, dass sie dank der Massnahme nun die Hilfe erhielten, die sie benötigten. Im Gegensatz zu früher bekämen sie schneller ein Spitalbett zugewiesen, wenn sie ein solches bräuchten [2].

Analog zur Einschätzung (einer Minderheit) der Patienten der Otago-Provinz steht eine Mehrheit der Psychiater Neuseelands ambulanten Zwangsbehandlungen grundsätzlich positiv gegenüber, betrachten diese aber nicht als eine Alternative zu guten gemeindenahen Angeboten, sondern - im Gegenteil - ganz von diesen abhängig [8]. Allerdings können ambulante Zwangsmassnahmen das Image der Psychiatrie beeinträchtigen, indem sie das Potential besitzen, Patienten von der Inanspruchnahme psychiatrischer Hilfe abzuhalten. Dies gilt für alle Personen, speziell aber für Patienten, die in ihrer Vorgeschichte bereits mit mehreren Formen von Zwangsmassnahmen in Berührung gekommen waren [9]. So weit erkennbar, stehen zwar sowohl Patienten als auch Angehörige und Therapeuten ambulanten Zwangsbehandlungen teilweise positiv gegenüber, äussern aber auch Kritik und Vorbehalte, so dass insgesamt eine ambivalente Situation besteht [10].

Zusammenfassend lässt sich festhalten, dass die zugängliche Literatur trotz zwei randomisierten kon- misierten kontrollierten Studien Patienten mit gewalttätigem Verhalten nicht zugelassen. Es ist daher aufgrund der verfügbaren Literatur nicht auszuschliessen, dass einzelne Patienten und die Gemeinschaft von ambulanten Zwangsmassnahmen profitieren können.

\section{Kritische Argumente}

Die institutionelle Psychiatrie geniesst in unserer Gesellschaft bis heute einen zwiespältigen Ruf, und unter den medizinischen Fachgebieten rangiert sie im untersten Bereich der Ansehens- und Einkommensskala. Sowohl psychisch kranke Menschen als auch Therapeuten sind mit Vorurteilen und Stigmatisierung konfrontiert. Psychiatrische «Anstalten» wurden während langer Zeit nicht nur als Orte von Therapie und Genesung, sondern auch als willige Vollzugsorgane eines repressiven Staates angesehen. Leider hat die Psychiatrie als Institution ihren Teil zu diesem Bild beigetragen, meist in unkritischer Erfüllung der ihr von der Gesellschaft zugewiesenen Rolle. Zu erwähnen sind beispielsweise das Mitwirken bei der Durchführung von Eugenik- und Sterilisationsprogrammen [12], bei der Diskriminierung afrikanischer Menschen [13], bei der Wegschliessung von Regimekritikern, bei der Langzeitinternierung von vermeintlich asozialen Personen und Unangepassten, wobei das eigene oder anderen empfohlene Handeln nicht selten mit «wissenschaftlichen Erkenntnissen» begründet wurde. Die Abseitsstellung der Psychiatrie innerhalb der Humanmedizin machte sich auch in Kleinigkeiten bemerkbar. Bis Ende 2004 war es beispielsweise im Kanton Zürich nicht möglich, ohne ein ärztliches Einweisungszeugnis freiwillig zur Erstbehandlung in eine 
psychiatrische Klinik einzutreten. Die Beschränkung wurde erst mit Inkrafttreten des neuen Patientengesetzes im Jahr 2005 aufgehoben - allerdings so beiläufig, dass die Neuregelung nicht einmal den Fachpersonen bekannt wurde.

Nach dem Zweiten Weltkrieg wurde die institutionelle Psychiatrie mit ihren überdimensionierten, abweisenden Einrichtungen in den Industrienationen einer fundamentalen Kritik unterworfen. Es kam zu Klinikschliessungen und ab dem Ende der 60er Jahre

\section{Patienten gaben an, dass sie dank der Zwangsmass- nahmen nun die Hilfe erhielten, die sie benötigten}

zum Aufbau von alternativen gemeindenahen Strukturen, die wir in ihrer Vielfalt heute als selbstverständlich gewordene Angebote vorfinden. Diese Entwicklung wurde durch die Stärkung der Rechtsstellung der Patienten, durch gerichtliche Kontrollen bei fürsorgerischen Freiheitsentziehungen und durch die Ent-

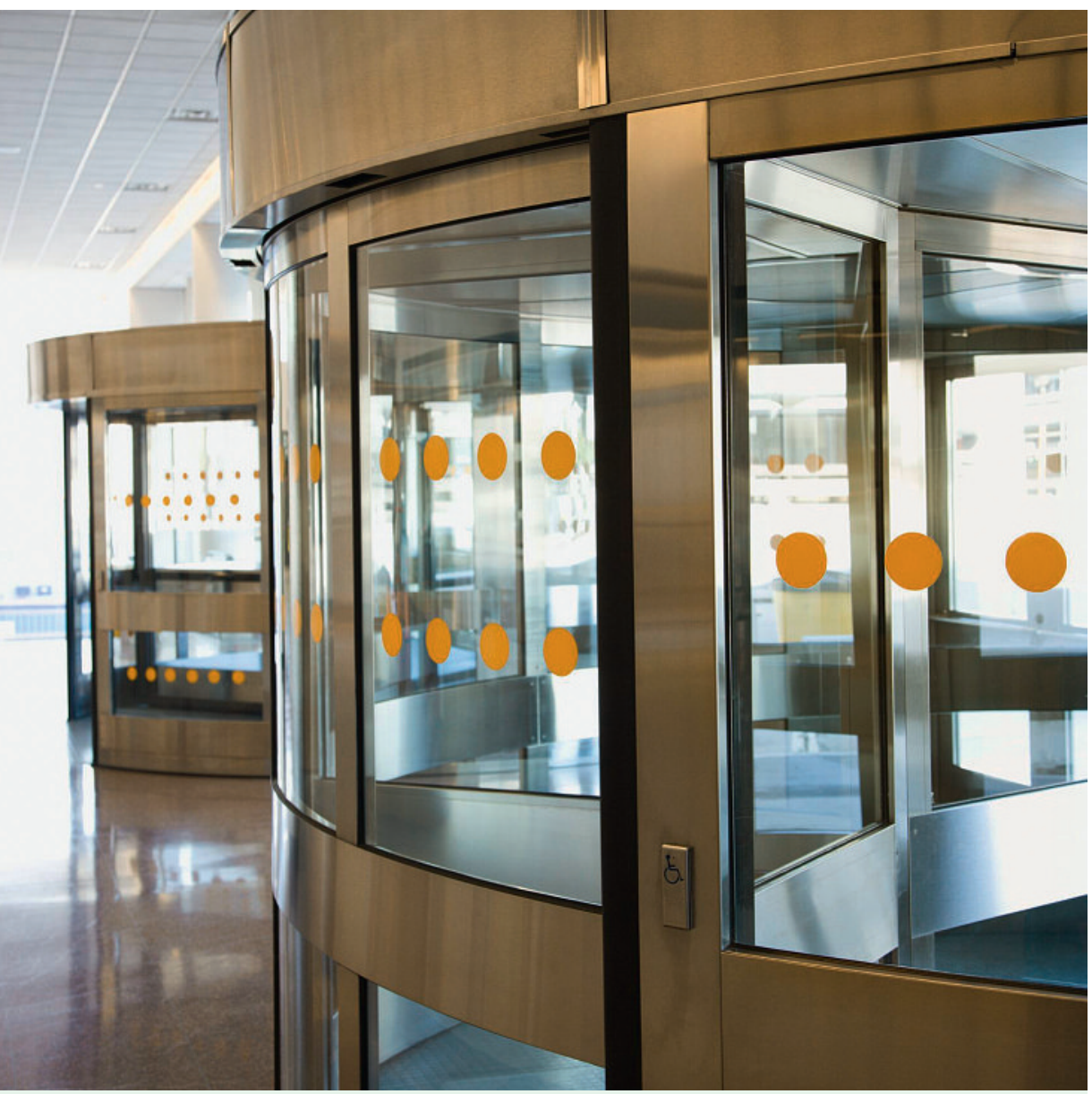

Die Effekte einer «Drehtürpsychiatrie» können durch ambulante psychiatrische Zwangsmassnahmen nicht abgeschwächt werden. wicklung von Ethikleitlinien stark gefördert. Jüngste Entwicklungen sind die Verankerung der Patientenverfügung im neuen Erwachsenenschutzrecht und das weltweite Anwachsen der Betroffenenbewegung, die der Psychiatrie die Definitionshoheit zum Begriff «Recovery» strittig macht und einen umfassenden Einbezug der Nutzerperspektive in die Psychiatrieplanung anstrebt.

Vor dem Hintergrund dieser Entwicklungen kann die Absicht, ambulante psychiatrische Zwangsmassnahmen in der Schweiz neu zuzulassen, als fragwürdige, rückwärtsgewandte Massnahme aufgefasst werden. Eine Erweiterung des Behandlungsrepertoires um ausserstationäre Zwangsmöglichkeiten mag die Befürchtung wecken, dass in Zukunft menschliche Zuwendung, therapeutische Beziehungsarbeit und argumentative Auseinandersetzung an Bedeutung verlieren und durch ein Mehr an Kontrolle, Zwang und Drohung ersetzt werden. Auf diese Weise würde einer eigentlichen Dehumanisierung in der Psychiatrie Vorschub geleistet, in einem medizinischen Fachgebiet also, das ganz besonders auf motivationale Klärung und behutsame mitmenschliche Begleitung angewiesen ist. An die Stelle eines durchaus kontroversen Diskurses zur Erarbeitung einer informierten Zustimmung (Informed consent) träte eher wieder ein mit Begriffen wie «Compliance» und «Krankheitseinsicht» operierendes, tendenziell paternalistisch geprägtes Krankheitsverständnis - möglicherweise verbunden mit der erhöhten Bereitschaft, bei Patienten, die eine evidenzbasierte Therapie ablehnen, a priori Urteilsunfähigkeit zu vermuten.

Zudem besteht die Gefahr, dass ambulanter Zwang als vermeintlich kostengünstige Alternative den längst fälligen Aufbau von personalintensiven Strukturen zur gemeindenahen Intensivbehandlung wie Akuttageskliniken oder aufsuchende mobile Teams verhindert oder zumindest den Entwicklungsdruck in diese Richtung abschwächt. Schliesslich können Zwangsmassnahmen die Beziehungsgestaltung zwischen Professionellen und Hilfesuchenden grundsätzlich erschweren und Menschen mit einer schweren psychischen Erkrankung von einer Inanspruchnahme der Angebote von sozialpsychiatrischen Einrichtungen abhalten [9].

\section{Schlussfolgerungen}

Die spezielle Geschichte der Psychiatrie und die mehrheitlich negativen Studienresultate ergeben keine überzeugende Evidenz für die Befürwortung von ambulanten psychiatrischen Zwangsmassnahmen. Insbesondere kann von Zwangsmassnahmen nicht generell erwartet werden, dass sie den Gesundheitszustand der Patienten verbessern, die Behandlungszufriedenheit steigern, Effekte einer «Drehtürpsychiatrie» abschwächen oder beispielsweise Verhaftungen bzw. Polizeikontakte verringern. Dennoch mag es Kantone geben, die ambulante Zwangsmassnahmen bei schwer psychisch kranken Menschen in begründeten Ausnahme- 
fällen als sinnvoll erachten und entsprechende Rechtsgrundlagen schaffen. Unseres Erachtens sollten die Massnahmen dann in erster Linie die Funktion haben, eine ineffektive Spirale mit wiederkehrendem Gefährdungspotenzial und Klinikeinweisungen in extremis (z. B. unter Zuzug der Polizei nach Aggressionshandlungen) zu unterbrechen - sei es durch ein tragfähiges ambulantes Setting oder durch eine rechtzeitige Klinikeinweisung. Eine Reduktion der Inanspruchnahme von externen Hilfestellungen oder von Spitalaufenthalten darf man sich davon aber - wie dargelegt nicht versprechen. Die Zwangsbehandlung sollte als zeitlich befristete Weisung an die betroffene Person ausgestaltet werden, sich in ärztliche Behandlung zu begeben oder sich einer bestimmten medikamentösen Behandlung zu unterziehen. Auf diese Weise gestattet sie eine engmaschige Begleitung der betroffenen Person und lässt es zu, rechtzeitig eine Fürsorgerische Unterbringung zu veranlassen, sofern die entsprechenden rechtlichen Voraussetzungen erfüllt sind. Auf Sanktionsmöglichkeiten wie eine polizeiliche Zuführung in ein psychiatrisches Ambulatorium oder eine Verabreichung von Medikamenten mittels körperlichen Zwangs ist gänzlich zu verzichten. Eine unmittelbare Durchsetzung von Massnahmen wie beispielsweise eine Zwangsmedikation im ambulanten Setting ist unseres Erachtens nicht praktikabel (s. auch die Botschaft des Bundesrates [3]) und wäre aufgrund einer Vielzahl von möglichen negativen Auswirkungen auf die betroffenen Personen und die psychiatrische Versorgung insgesamt als kontraproduktiv einzustufen.

\section{Literatur}

(gültig für Teil 1 und 2)

1 O'Brien AJ, McKenna BG, Kydd RR. Compulsory community mental health treatment: Literature review. International Journal of Nursing Studies. 2009; 46:1245-55.

2 Gibbs A, Dawson J, Ansley C, Mullen R. How patients in New Zealand view community treatment orders. Journal of Mental Health. 2005;14(4):357-68.
3 Botschaft des Bundesrates zur Änderung des Schweizerischen Zivilgesetzbuches (Erwachsenenschutz, Personenrecht und Kindesrecht) vom 28. Juni 2006. S. 7071

4 Churchill R, Owen G, Singh S, Hotopf M. International Experiences of community treatment orders. Institute of Psychiatry. London; 2007.

5 Burns T, Dawson J. Community treatment orders: how ethical without experimental evidence? Psychological Medicine. 2009;39:1583-6.

6 Kisely SR, Campbell LA, Preston NJ. Compulsory community and involuntary outpatient treatment for people with severe mental illness. Cochrane Database of Systematic Reviews. Issue 3; 2005.

7 Kisely SR, Campbell LA, Scott A, Preston NJ, Xiao J. Randomized and non-randomized evidence for the effect of compulsory community and involuntary out-patient treatment on health service use: systematic review and meta-analysis. Psychological Medicine. 2007;37:3-14.

8 Romans S, Dawson J, Mullen R, Gibbs A. How mental health clinicians view community treatment orders: a national New Zealand survey. Australian and New Zealand Journal of Psychiatry. 2004;38:836-41.

9 Van Dorn RA, Elbogen EB, Redlich AD, Swanson JW, Swartz MS, Mustillo S. The relationship between mandatet community treatment and perceived barriers to care in persons with severe mental illness. International Journal of Law and Psychiatry. 2006;29:495-506.

10 Dawson J, Romans S, Gibbs A, Ratter N. Ambivalence about community treatment orders. International Journal of Law and Psychiatry. 2003;26:243-55.

11 Kisely S, Campbell LA. Does compulsory or supervised community treatment reduce «revolving door» care? British Journal of Psychiatry. 2007;191:373-4.

12 Imboden G, Ritter HJ, Braunschweig S, Küchenhoff B, Wecker R. In: Wecker R, Braunschweig S, Imboden G, Küchenhoff B, Ritter HJ (Hrsg.). Wie nationalsozialistisch ist die Eugenik? Wien, Köln, Weimar: Böhlau Verlag; 2009. S.13-21.

13 Fanon F. Die Verdammten dieser Erde. Frankfurt am Main: Suhrkamp Verlag; 1981. 